\title{
Actitudes hacia las matemáticas en los estudiantes del 7 mo a 9no de educación básica de Honduras
}

\author{
Carlos Alberto Gutiérrez Perdomo ${ }^{1}$ \\ Orlando Castro Hernández ${ }^{2}$ \\ Ronald Xavier Hernández Carrasco ${ }^{3}$
}

\section{Información de artículo:}

Recibido: 30/03/2021

Aprobado:30/05/2021

\section{Palabras claves:}

Actitudes, agrado, ansiedad, confianza, motivación, utilidad.

\section{Key Words:}

.Attitudes, Liking, Anxiety, Confidence, Motivation, Usefulness.

\section{Resumen}

En esta investigación se ha analizado las actitudes que los estudiantes del tercer ciclo de educación básica presentan hacia las matemáticas. Es un estudio cuantitativo con un diseño "Ex Post Facto" a partir de la aplicación de una escala de actitud hacia las matemáticas a una muestra de 247 estudiantes de tres departamentos de Honduras: Lempira, La Paz y El Paraíso. Se realizó un análisis descriptivo e inferencial de los datos con ayuda del Sistema Estadística para las Ciencias Sociales. Los resultados muestran que los estudiantes poseen una actitud positiva hacia las matemáticas, principalmente en los factores confianza, utilidad y agrado. Cabe destacar que el nivel de ansiedad es bajo, sin embargo, los estudiantes se encuentran desmotivados. Los factores con la correlación más alta son confianza-agrado, lo que significa que los estudiantes se sienten bien cuando están aprendiendo matemáticas y provoca satisfacción resolver problemas matemáticos. Es importante mencionar que cuando los factores confianza y agrado se correlacionan con la motivación, se obtiene un coeficiente negativo, lo que conduce a resultados inversamente proporcionales. La desmotivación conlleva a disminuir el agrado y la confianza. Se concluye que en las variables cualitativas género, lugar de procedencia y dispositivos inteligentes para conexión a clases virtuales utilizados por los estudiantes, no presentan diferencias significativas de la actitud hacia las matemáticas. Igualmente, los estudiantes pertenecientes a los grados séptimo, octavo y noveno tienen el mismo nivel de confianza y desmotivación; y el séptimo grado tiene mayor agrado y utilidad, y menor ansiedad.

\footnotetext{
1 Profesorado en Matemáticas en el grado de Licenciatura, Profesor de matemáticas en Centro de Educación Básica Marco Aurelio Soto. E-mail: math7albert@gmail.com (iD) https://orcid.org/0000-0003-1244-0831

2 Profesorado en Matemáticas en el grado de Licenciatura. Profesor de matemáticas en Centro de Educación Básica Manuel Bonilla. E-mail: orlandoh118@gmail.com (iD) https://orcid.org/oooo-0003-4522-3112

3 Profesorado en Matemáticas en el grado de Licenciatura. Profesor de matemáticas en el Instituto Polivalente Dr. Presentación Centeno. E-mail: xavier_3808@hotmail.com (D): https://orcid.org/oooo-0001-8325-9779
} 


\section{Attitudes towards mathematics in students from 7th to 9th of basic education in Honduras}

\section{Abstract}

This research has analyzed the attitudes that students of the third cycle of basic education have towards mathematics. It is a quantitative study with a descriptive design based on the application of an attitude towards mathematics scale to a sample of 247 students from three departments in Honduras: Lempira, La Paz and El Paraíso. A descriptive and inferential analysis of the data was carried out with the help of the Statistical System for the Social Sciences. The results show that students have a positive attitude towards mathematics, mainly in the factors of trust, usefulness and liking. It should be noted that the level of anxiety is low, however, the students are unmotivated. The factors with the highest correlation are confidence-liking, which means that students feel good about learning math and solving math problems is satisfying. It is important to mention that when the trust and liking factors are correlated with motivation, a negative coefficient is obtained, which leads to inversely proportional results. Demotivation leads to a decrease in liking and confidence. It is concluded that in the qualitative variables gender, place of origin and smart devices for connection to virtual classes used by students, do not present significant differences in attitude towards mathematics. Likewise, students belonging to the seventh, eighth and ninth grades have the same level of confidence and demotivation; and the seventh grade has greater enjoyment and usefulness, and less anxiety.

\section{Introducción}

En el campo educativo con frecuencia se escuchan expresiones de los estudiantes como: No me gustan las matemáticas, no soy bueno con las matemáticas, las matemáticas no son divertidas ni tienen alguna utilidad. Por lo que se denota un claro desinterés. En ese sentido, "el estudio de las actitudes hacia las matemáticas es fundamental porque impiden [las actitudes] una progresión en el aprendizaje de las matemáticas" (Flores \& Auzmendi, 2018, p. 232). Este obstáculo aumenta a medida que se van estudiando nuevos temas y avanzando en los grados académicos.

Por lo expuesto se decide realizar una investigación con estudiantes del tercer ciclo de educación básica (séptimo, octavo y noveno grado), utilizando como técnica de recolección de datos Formularios de Google. El propósito de esta investigación es analizar las actitudes hacia las matemáticas por parte de los estudiantes del tercer ciclo de educación básica de los departamentos de Lempira, La Paz y El Paraíso, aplicando la escala de actitudes hacia las matemáticas de Auzmendi (1992), en la cual se identifican cinco factores actitudinales: ansiedad, agrado, utilidad, motivación y confianza. El estudio tiene como objetivo general determinar las actitudes de los estudiantes hacia 
las matemáticas, tomando en consideración las variables género, grado académico, tipo de dispositivo de conectividad y lugar de procedencia de los estudiantes.

\section{Literatura}

La actitud es el comportamiento que se produce en diferentes circunstancias. Estas determinan el estado de ánimo de cada individuo y se van fortaleciendo de acuerdo con las acciones repetidas (Chamorro-Sady, Pérez-Henríquez \& Flores, 2018). La formación de actitudes favorece el equilibrio de la persona, además permite afrontar positiva o negativamente una situación. En el proceso de formación, los estudiantes reflejan diferentes comportamientos antes las asignaturas que cursan. En matemáticas es donde normalmente se presentan diferencias notorias en cuanto a la actitud; ya sea por la valoración y aprecio hacia la asignatura o el interés por asimilar los contenidos.

La actitud hacia las matemáticas se refiere a la valoración, el aprecio, la satisfacción, la curiosidad y el interés tanto por la disciplina como por su aprendizaje, acentuando más el componente afectivo que el cognitivo (Flores y Auzmendi, 2015). En ese sentido, es un aspecto de sumo interés para la investigación ya que permite conocer como se sienten los estudiantes hacia esta asignatura y así poder tomar decisiones que vayan encaminadas a diseñar estrategias didácticas, formas de evaluación y personalizar la atención que reciben los estudiantes.

Las actitudes en educación se han definido ya a lo largo del siglo pasado de diversas formas. Así lo manifiesta MacLeod (1989), citado por Fernández-Cézar y Aguirre-Pérez (2010) que todas las definiciones de Aiken (1970) y Hart (1989) sobre actitud incluyen un elemento comportamental, es decir, que las actitudes actúan como una fuerza motivacional del comportamiento humano. Pero en lo relativo al concepto de actitud en educación matemática, los educadores han usado el término con una definición menos clara que los psicólogos, como algo observable a través de instrumentos de medida, diseñados para evaluar los componentes específicos de la actitud (pp. 107-108).

Algunos instrumentos de medida como los cuestionarios se centran en uno o varios aspectos relacionados con la actitud, con el propósito de medir alguna característica específica que conviene conocer y analizar. Dentro de estos instrumentos se encuentra la Escala de Actitud hacia las Matemáticas (EAM) desarrollada por Auzmendi (1992); la cual es una encuesta de auto reporte, anónima, multidimensional, compuesta por cinco factores los cuales forman las subescalas que miden la ansiedad, el agrado, la utilidad, la motivación y la confianza hacia las matemáticas. Estos factores están organizados en veinticinco ítems que ilustran diferentes aspectos afectivos. A continuación, se definen cada uno de estos factores (Flores \& Auzmendi, 2018):

- Agrado, hace referencia al aspecto de agrado o disfrute que provoca el trabajo matemático. 
- Ansiedad, se refiere al sentimiento de ansiedad, temor que el estudiante manifiesta ante la materia de matemáticas.

- Motivación, puede interpretarse como la motivación que siente el estudiante hacia el estudio y utilización de las matemáticas.

- Utilidad, hace referencia al valor que el estudiante otorga a las matemáticas, a la utilidad que él percibe que puede tener esta materia para su futura vida profesional.

- Confianza, puede interpretarse como el sentimiento de confianza que provoca la habilidad en matemáticas.

\section{Metodología de investigación}

\subsection{Finalidad y objetivos del estudio}

Esta investigación tiene por objetivo general determinar las actitudes de los estudiantes hacia las matemáticas, tomando en consideración las variables género, lugar de procedencia, uso de dispositivos tecnológicos y grado académico, por tales razones, se proponen los objetivos específicos siguientes: (1) Identificar las actitudes hacia las matemáticas; (2) Explicar la relación entre los factores actitudinales hacia las matemáticas; (3) Comprobar si existen diferencias significativas entre las variables género, lugar de procedencia, uso de dispositivos tecnológicos y grado académico en relación con las actitudes hacia las matemáticas.

\subsection{Metodología de la investigación}

El enfoque de esta investigación es cuantitativo. Este paradigma ha guiado el tratamiento de los datos a través de la categorización y descripción de las propiedades, características y perfiles de las personas, grupos, comunidades, procesos y objetos que se han sometidos a análisis (Hernández-Sampieri, Fernández-Collado, Baptista-Lucio, 2014, pp. 4-6). El diseño de esta investigación es "Ex Post Facto" porque este tipo de investigación trata de descubrir fenómenos que ocurren en forma natural, pero miden diversas variables para analizar su posible efecto (Bisquerra, 2012).

\subsection{Descripción de los participantes}

El estudio se llevó a cabo con una muestra de 247 estudiantes de tercer ciclo de educación básica, pertenecientes a 3 departamentos de Honduras: Lempira (57\%), La Paz (5\%) y El Paraíso (38\%). 


\begin{tabular}{|l|c|c|c|}
\hline \multicolumn{4}{|c|}{ Tabla 1. Participantes de la Investigación } \\
\hline Grado & Mujeres & Hombres & Total \\
\hline Séptimo & 48 & 46 & 94 \\
\hline Octavo & 42 & 28 & 70 \\
\hline Noveno & 55 & 28 & 83 \\
\hline Total & 145 & 102 & 247 \\
\hline
\end{tabular}

El 59\% (145) de los participantes son mujeres, frente al 41\% (102) que son hombres. Respecto a su edad, la media se sitúa en 13 años, encontrándose el 95.20\% de los participantes en el intervalo de edad entre 11-15 años. En referencia a la variable grado académico, el 38\% son de séptimo grado; $28 \%$ son de octavo grado; y $34 \%$ de noveno grado. Otra característica de la muestra es que la mayoría de los estudiantes utilizan tecnologías de la información y comunicación para acceder a sus estudios virtuales. Finalmente, hay que indicar que el tipo de muestreo que hemos utilizado ha sido el muestreo no probabilístico causal o accidental, en el cual el investigador selecciona directa e intencionalmente la muestra, debido fundamentalmente, a que tiene fácil acceso a la misma y es representativa de la población (Gil, Rodríguez y García, 1995; Albert-Gómez, 2007; Sabariego, 2004).

\subsection{Instrumentos de recogida de datos}

El instrumento para la recopilación de la información fue el cuestionario de actitudes hacia las matemáticas de Auzmendi (1992). Según Flores y Auzmendi (2018) el instrumento está constituido por 25 ítems, aglutinados en 5 factores asociados a los diferentes componentes de las actitudes hacia las matemáticas. Los estudiantes pueden indicar su agrado de acuerdo o desacuerdo con las afirmaciones propuestas por media de una escala Likert de 5 valores. Los factores asociados son: agrado, ansiedad, motivación, utilidad y confianza.

\subsection{Procedimiento de administración y enfoque ético}

La administración del instrumento se realizó por parte de los autores durante el primer semestre de 2021, tenía un carácter anónimo y fue completado por los sujetos participantes mediante Formularios de Google en presencia del profesorado. Con anterioridad a la toma de datos, se obtuvo tanto el consentimiento previo, libre e informado de los participantes, así como la aprobación de las autoridades educativas.

\subsection{Análisis de datos}

Para satisfacer el objetivo del estudio se procedió al desarrollo de distintos análisis, empleando el paquete estadístico para las ciencias sociales SPSS v.24. Entre ellos, se aplicó un análisis de consistencia interna, un análisis descriptivo de los datos, un análisis de las correlaciones entre las categorías y pruebas paramétricas y no paramétricas. 


\section{Resultados}

Los resultados reflejan una media de 88.89 puntos $(S D=84.00)$ por lo que se puede decir que los estudiantes manifiestan una actitud global positiva hacia las matemáticas, ubicándose la mayoría en una moda de 84 puntos.

\subsection{Actitudes hacia las matemáticas}

En el factor ansiedad tiene un máximo de 45 puntos y un mínimo de 9 puntos, los participantes presentaron una media de 31.27 puntos $(S D=5.91)$, con una varianza de $35.02 \%$. Considerando que la escala está codificada de modo que a mayor puntuación menor ansiedad (Flores y Auzmendi, 2015) se concluye que el nivel de ansiedad en los estudiantes es moderadamente bajo con respecto a la media. El ítem 8 (Tengo confianza en mí mismo/a cuando me enfrento a un problema de matemáticas) presenta la mayor puntuación, esto significa que los estudiantes sienten poca ansiedad cuando se enfrentan a un problema de matemáticas.

\begin{tabular}{|l|c|c|c|c|c|c|}
\hline \multicolumn{7}{|c|}{ Tabla 2. Ansiedad hacia las matemáticas } \\
\cline { 2 - 7 } Criterios & $\begin{array}{c}\text { Fuerte- } \\
\text { mente en } \\
\text { desacu- } \\
\text { erdo }\end{array}$ & $\begin{array}{c}\text { En } \\
\text { desacu- } \\
\text { erdo }\end{array}$ & $\begin{array}{c}\text { Indife- } \\
\text { rente }\end{array}$ & $\begin{array}{c}\text { De } \\
\text { acuerdo }\end{array}$ & $\begin{array}{c}\text { Fuerte- } \\
\text { mente } \\
\text { de acu- } \\
\text { erdo }\end{array}$ & $\begin{array}{c}\text { Puntu- } \\
\text { dián (SD) }\end{array}$ \\
\hline $\begin{array}{l}\text { 2. La asignatura de matemáticas } \\
\text { se me da bastante mal. }\end{array}$ & 5 & 14 & 26 & 26 & 29 & $3.60(1.17)$ \\
\hline $\begin{array}{l}\text { 3. Estudiar o trabajar con las } \\
\text { matemáticas no me asustan en } \\
\text { absoluto. }\end{array}$ & 14 & 12 & 13 & 37 & 24 & $3.46(1.34)$ \\
\hline $\begin{array}{l}\text { 7. Las matemáticas es una de las } \\
\text { asignaturas que más temo. }\end{array}$ & 10 & 21 & 17 & 30 & 22 & $3.31(1.30)$ \\
\hline $\begin{array}{l}\text { 8. Tengo confianza en mín mismo/a } \\
\text { cuando me enfrento a un prob- } \\
\text { lema de matemáticas. }\end{array}$ & 3 & 6 & 12 & 39 & 40 & $4.06(1.01)$ \\
\hline $\begin{array}{l}\text { 12. Cuando me enfrento a un } \\
\text { problema de matemática me sien- } \\
\text { to incapaz de pensar con claridad. }\end{array}$ & 13 & 23 & 15 & 30 & 19 & $3.18(1.32)$ \\
\hline $\begin{array}{l}\text { 13. Estoy calmado/a y tranquilo/a } \\
\text { cuando me enfrento a un prob- } \\
\text { lema de matemáticas. }\end{array}$ & 5 & 12 & 13 & 43 & 27 & $3.76(1.12)$ \\
\hline $\begin{array}{l}\text { 17. Trabajar con las matemáticas } \\
\text { hace que me sienta nervioso/a. }\end{array}$ & 11 & 30 & 15 & 30 & 14 & $3.07(1.26)$ \\
\hline $\begin{array}{l}\text { 18. No me altero cuando tengo } \\
\text { que trabajar en problema de } \\
\text { matemáticas. }\end{array}$ & 9 & 15 & 16 & 40 & 20 & $3.46(1.23)$ \\
\hline $\begin{array}{l}\text { 22. Las matemáticas hacen que me } \\
\text { sientan incómodo/a y nervioso/a. }\end{array}$ & 8 & 21 & 17 & 35 & 19 & $3.34(1.22)$ \\
\hline Puntuación Total a & & & & & & \\
\hline
\end{tabular}




\begin{tabular}{|c|c|c|c|c|c|c|}
\hline \multirow[b]{2}{*}{ Criterios } & \multicolumn{5}{|c|}{ Respuestas \% } & \multirow[b]{2}{*}{$\begin{array}{l}\text { Puntu- } \\
\text { ación Me- } \\
\text { dia (SD) }\end{array}$} \\
\hline & $\begin{array}{c}\text { Fuerte- } \\
\text { mente en } \\
\text { desacu- } \\
\text { erdo }\end{array}$ & $\begin{array}{c}\text { En } \\
\text { desacu- } \\
\text { erdo }\end{array}$ & $\begin{array}{l}\text { Indife- } \\
\text { rente }\end{array}$ & $\begin{array}{c}\text { De } \\
\text { acuerdo }\end{array}$ & $\begin{array}{c}\text { Fuerte- } \\
\text { mente } \\
\text { de acu- } \\
\text { erdo }\end{array}$ & \\
\hline Mediana (rango) & \multicolumn{6}{|l|}{$32(13-45)$} \\
\hline Media (SD) & \multicolumn{6}{|l|}{$31.27(5.91)$} \\
\hline Varianza & \multicolumn{6}{|l|}{35.02} \\
\hline Puntuación total \% & \multicolumn{6}{|l|}{$69 \%$} \\
\hline
\end{tabular}

En el factor agrado se presenta una puntuación media de los estudiantes de 14. $24(\mathrm{SD}=3.41)$ de un máximo de 20 puntos, con una varianza de $11.65 \%$. Se concluye que los estudiantes del tercer ciclo de educación básica demuestran agrado por las matemáticas, incluso estarían dispuestos a inscribirse en más cursos de matemáticas de los que son obligatorios (ítems 25), además de que les resulta divertida.

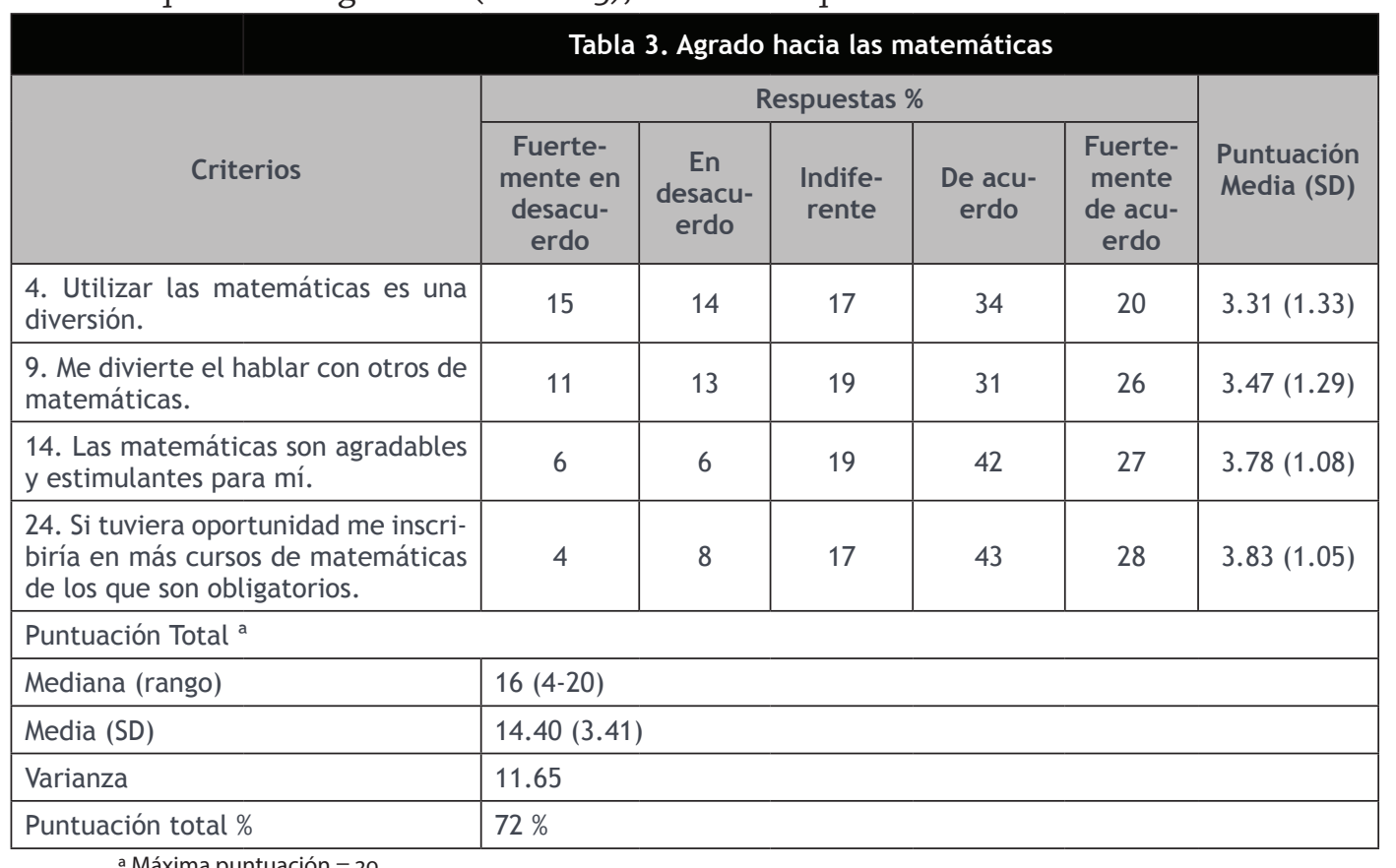

a Máxima puntuación $=20$

Con un rango de puntuación de 6-30 puntos mínimo y máximo respectivamente, la media de la muestra es de 21.91 puntos $(S D=3.59)$. Los estudiantes reconocen que las matemáticas tienen utilidad en sus estudios y la vida profesional. Claramente se evidencia en los ítems (1, 6, 21 y 19) con puntuación más alta. A pesar de considerarla útil, en los ítems ( $15 \mathrm{y} 16)$ con menor puntuación manifiestan que hay otras asignaturas más importantes para su futura profesión y esperan no utilizarla en su vida profesional. 


\begin{tabular}{|c|c|c|c|c|c|c|}
\hline \multirow[b]{2}{*}{ Criterios } & \multicolumn{5}{|c|}{ Respuestas \% } & \multirow[b]{2}{*}{$\begin{array}{l}\text { Puntuación } \\
\text { Media (SD) }\end{array}$} \\
\hline & $\begin{array}{l}\text { Fuerte- } \\
\text { mente en } \\
\text { desacu- } \\
\text { erdo }\end{array}$ & $\begin{array}{l}\text { En } \\
\text { desacu- } \\
\text { erdo }\end{array}$ & $\begin{array}{l}\text { Indife- } \\
\text { rente }\end{array}$ & $\begin{array}{l}\text { De acu- } \\
\text { erdo }\end{array}$ & $\begin{array}{l}\text { Fuerte- } \\
\text { mente de } \\
\text { acuerdo }\end{array}$ & \\
\hline $\begin{array}{l}\text { 1. Considero las matemáticas como una } \\
\text { materia muy necesaria en mis estudios. }\end{array}$ & 2 & 1 & 5 & 23 & 69 & $4.57(0.77)$ \\
\hline $\begin{array}{l}\text { 6. Quiero llegar a tener un conocimien- } \\
\text { to más profundo de las matemáticas. }\end{array}$ & 4 & 1 & 5 & 33 & 57 & $4.37(0.94)$ \\
\hline $\begin{array}{l}\text { 15. Espero tener que utilizar poco las } \\
\text { matemáticas en mi vida profesional. }\end{array}$ & 18 & 32 & 16 & 21 & 13 & $2.80(1.32)$ \\
\hline $\begin{array}{l}\text { 16. Considero que existen otras asig- } \\
\text { naturas más importantes que las } \\
\text { matemáticas para mi futura profesión. }\end{array}$ & 18 & 29 & 23 & 18 & 12 & $2.76(1.27)$ \\
\hline $\begin{array}{l}\text { 19. Me gustaría tener una ocupa- } \\
\text { ción en la cual tuviera que utilizar las } \\
\text { matemáticas. }\end{array}$ & 10 & 15 & 19 & 35 & 21 & $3.42(1.24)$ \\
\hline $\begin{array}{l}\text { 21. Para mi futuro profesional la } \\
\text { matemática es una de las asignaturas } \\
\text { más importantes que tengo que estu- } \\
\text { diar. }\end{array}$ & 4 & 7 & 12 & 39 & 38 & $3.97(1.09)$ \\
\hline \multicolumn{7}{|l|}{ Puntuación Total ${ }^{a}$} \\
\hline Mediana (rango) & \multicolumn{6}{|l|}{$18(12-30)$} \\
\hline Media (SD) & \multicolumn{6}{|l|}{ 21.91(3.59) } \\
\hline Varianza & \multicolumn{6}{|l|}{12.943} \\
\hline Puntuación total \% & \multicolumn{6}{|l|}{$73 \%$} \\
\hline
\end{tabular}

a Máxima puntuación $=30$

Con respecto al factor motivación, se presenta una media de 8.73 puntos $(\mathrm{SD}=5.02)$, lo que indica claramente que los estudiantes se encuentran desmotivados. Esto debido a que perciben los contenidos impartidos como poco interesantes (ítem 25), es demasiado teórica para que pueda servir de algo (ítem 5 ), además que las matemáticas son útiles para quienes estudiarán una carrera de ciencia (ítem 10). Muchos estudiantes optan por estudiar una carrera donde no tengan que estudiar matemáticas ya sea a nivel de educación media o superior.

\begin{tabular}{|c|c|c|c|c|c|c|}
\hline \multirow[b]{2}{*}{ Criterios } & \multicolumn{5}{|c|}{ Respuestas \% } & \multirow[b]{2}{*}{$\begin{array}{c}\text { Puntu- } \\
\text { ación } \\
\text { Media } \\
\text { (SD) }\end{array}$} \\
\hline & $\begin{array}{l}\text { Fuerte- } \\
\text { mente en } \\
\text { desacuerdo }\end{array}$ & $\begin{array}{l}\text { En de- } \\
\text { sacuerdo }\end{array}$ & $\begin{array}{l}\text { Indife- } \\
\text { rente }\end{array}$ & $\begin{array}{l}\text { De acu- } \\
\text { erdo }\end{array}$ & $\begin{array}{l}\text { Fuerte- } \\
\text { mente } \\
\text { de acu- } \\
\text { erdo }\end{array}$ & \\
\hline $\begin{array}{l}\text { 5. La matemática es demasi- } \\
\text { ada teórica para que pueda } \\
\text { servirme de algo. }\end{array}$ & 25 & 28 & 14 & 16 & 17 & $\begin{array}{c}2.72 \\
(1.43)\end{array}$ \\
\hline
\end{tabular}


Tabla 5. Motivación hacia las matemáticas

\begin{tabular}{|l|l|c|c|c|c|c|}
\hline \multirow{2}{*}{ Criterios } & \multicolumn{5}{|c|}{ Respuestas \% } & Puntu- \\
\cline { 2 - 6 } & $\begin{array}{c}\text { Fuerte- } \\
\text { mente en } \\
\text { desacuerdo }\end{array}$ & $\begin{array}{c}\text { En de- } \\
\text { sacuerdo }\end{array}$ & $\begin{array}{c}\text { Indife- } \\
\text { rente }\end{array}$ & $\begin{array}{c}\text { De acu- } \\
\text { erdo }\end{array}$ & $\begin{array}{c}\text { Fuerte- } \\
\text { mente } \\
\text { de acu- } \\
\text { erdo }\end{array}$ & $\begin{array}{c}\text { Medión } \\
\text { (SD) }\end{array}$ \\
\hline $\begin{array}{l}\text { 10. Las matemáticas pueden } \\
\text { ser útiles para el que decida } \\
\text { realizar una carrera de cien- } \\
\text { cias, pero no para el resto de } \\
\text { los estudiantes. }\end{array}$ & 26 & 27 & 19 & 16 & 12 & $\begin{array}{c}2.61 \\
(1.34)\end{array}$ \\
\hline $\begin{array}{l}\text { 25. La matemática que se } \\
\text { imparte en las clases de } \\
\text { matemáticas es muy poco in- } \\
\text { teresante. }\end{array}$ & 14 & 15 & 11 & 38 & 22 & 3.39 \\
\hline Puntuación Total a & $12(3-15)$ & & & & \\
\hline Mediana (rango) & $8.73(2.95)$ & & & & \\
\hline Media (SD) & 8.74 & & & & \\
\hline Varianza & $58.20 \%$ & & & & \\
\hline Puntuación total \%
\end{tabular}

a Máxima puntuación $=15$

El factor confianza presenta la puntuación más alta del instrumento, $83.66 \%$. Además, tiene una media de 12.55 puntos $(S D=2.18)$, lo que evidencia que los estudiantes sienten mucha confianza en las matemáticas; considerando de la menor a la mayor puntuación, a los estudiantes les genera gran satisfacción llegar a resolver un problema matemático (ítem 20) y, que si se lo proponen pueden llegar a dominarlas bien (ítem 23).

\begin{tabular}{|c|c|c|c|c|c|c|}
\hline \multirow[b]{2}{*}{ Criterios } & \multicolumn{5}{|c|}{ Respuestas \% } & \multirow[b]{2}{*}{$\begin{array}{l}\text { Puntu- } \\
\text { ación } \\
\text { Media (SD) }\end{array}$} \\
\hline & $\begin{array}{l}\text { Fuerte- } \\
\text { mente } \\
\text { en de- } \\
\text { sacuerdo }\end{array}$ & $\begin{array}{c}\text { En de- } \\
\text { sacuerdo }\end{array}$ & $\begin{array}{l}\text { Indife- } \\
\text { rente }\end{array}$ & $\begin{array}{l}\text { De acu- } \\
\text { erdo }\end{array}$ & $\begin{array}{l}\text { Fuerte- } \\
\text { mente de } \\
\text { acuerdo }\end{array}$ & \\
\hline $\begin{array}{l}\text { 11. Tener buenos cono- } \\
\text { cimientos de matemáticas } \\
\text { incrementará mis posibili- } \\
\text { dades de trabajo. }\end{array}$ & 2 & 1 & 9 & 36 & 52 & $4.35(0.84)$ \\
\hline $\begin{array}{l}\text { 20. Me provoca una gran sat- } \\
\text { isfacción el llegar a resolver } \\
\text { problemas de matemáticas. }\end{array}$ & 6 & 10 & 14 & 37 & 33 & $3.81(1.15)$ \\
\hline $\begin{array}{l}\text { 23. Si me lo propusiera creo } \\
\text { que llegaría a dominar bien } \\
\text { las matemáticas. }\end{array}$ & 2 & 1 & 5 & 39 & 53 & $4.38(0.82)$ \\
\hline \multicolumn{7}{|l|}{ Puntuación Total a } \\
\hline
\end{tabular}




\begin{tabular}{|c|c|c|c|c|c|c|}
\hline \multirow[b]{3}{*}{ Criterios } & \multicolumn{6}{|c|}{ Tabla 6. Confianza hacia las matemáticas } \\
\hline & \multicolumn{5}{|c|}{ Respuestas \% } & \multirow[b]{2}{*}{$\begin{array}{c}\text { Puntu- } \\
\text { ación } \\
\text { Media (SD) }\end{array}$} \\
\hline & $\begin{array}{l}\text { Fuerte- } \\
\text { mente } \\
\text { en de- } \\
\text { sacuerdo }\end{array}$ & $\begin{array}{l}\text { En de- } \\
\text { sacuerdo }\end{array}$ & $\begin{array}{l}\text { Indife- } \\
\text { rente }\end{array}$ & $\begin{array}{l}\text { De acu- } \\
\text { erdo }\end{array}$ & $\begin{array}{l}\text { Fuerte- } \\
\text { mente de } \\
\text { acuerdo }\end{array}$ & \\
\hline Mediana (rango) & \multicolumn{6}{|l|}{$12(3-15)$} \\
\hline Media (SD) & \multicolumn{6}{|c|}{$12.55(2.18)$} \\
\hline Varianza & \multicolumn{6}{|l|}{4.77} \\
\hline Puntuación total \% & \multicolumn{6}{|l|}{$83.66 \%$} \\
\hline
\end{tabular}

a Máxima puntuación $=15$

\subsection{Relación entre los factores actitudinales hacia las matemáticas}

La relación ansiedad-agrado se correlacionan positivamente con un $r$-Pearson de 0.459. Dado que los estudiantes presentan una puntuación baja en el factor ansiedad, esta correlación significa que a menor ansiedad hay mayor agrado y a la inversa, a menor agrado mayor ansiedad. En cuanto a los factores agrado-utilidad se correlacionan positivamente, entonces son directamente proporcional. Analizando el r-Pearson o.449, se concluye que, a mayor agrado hacia las matemáticas mayor utilidad, o cuando disminuye el agrado disminuye la utilidad hacia las matemáticas y viceversa. Los estudiantes perciben que las matemáticas son útiles para la vida.

\begin{tabular}{|c|c|c|c|c|c|}
\hline & \multicolumn{5}{|c|}{ Tabla 7: Análisis de correlaciones entre las categorías ( $r$-Pearson) } \\
\hline Factores & Ansiedad & Agrado & Utilidad & Motivación & Confianza \\
\hline Ansiedad & 1 & 0.456 & 0.516 & 0.341 & 0.296 \\
\hline Agrado & & 1 & 0.449 & -0.152 & 0.489 \\
\hline Utilidad & & & 1 & 0.199 & 0.457 \\
\hline Motivación & & & & 1 & -0.034 \\
\hline Confianza & & & & & 1 \\
\hline
\end{tabular}

La correlación $r$-Pearson de la relación motivación-agrado es de -0.152, un coeficiente negativo, indicándonos que son factores inversamente proporcionales. Al crecer el agrado disminuye a motivación, así se aprecia en la tabla 3 y tabla 5; a los estudiantes les agrada las matemáticas, pero se sienten desmotivados. Del mismo modo sucede con un coeficiente $r$-Pearson de -0.034 cuando se relaciona motivación-confianza, a pesar de que los estudiantes se sienten en confianza esta disminuye porque no están motivados. El factor confianza es el que tiene mayor puntuación en el instrumento, este tiene una correlación $r$-Pearson de 0.489 y 0.457 al relacionarlo con los factores 
agrado y utilidad. Ambas relaciones son positivas, por lo tanto, los estudiantes sienten agrado hacia las matemáticas y encuentran su utilidad en sus estudios y vida laboral. El resto de las correlaciones de los factores actitudinales son positivas, aunque no son tan significativas.

\subsection{Variables que determinan las actitudes hacia las matemáticas}

Aplicando una prueba de normalidad de Kolmogorov-Smirnov para una muestra se decide que los factores agrado, utilidad, motivación y confianza son significativas, es decir que son menores que 0.05 por lo tanto se les aplica pruebas no paramétricas y al otro factor, ansiedad, que es de 0.070 se le aplica una prueba paramétrica.

\begin{tabular}{|l|c|c|c|c|}
\hline \multicolumn{5}{|c|}{ Tabla 8: Análisis paramétrico o no paramétrico } \\
\hline \multirow{2}{*}{ Factores } & Género & Grado & $\begin{array}{c}\text { Lugar de pro- } \\
\text { cedencia }\end{array}$ & Dispositivo \\
\cline { 2 - 5 } & $p$ & $p$ & $p$ & $p$ \\
\hline Ansiedad & 0.060 & 0.001 & 0.120 & 0.315 \\
\hline Agrado & 0.486 & 0.027 & 0.402 & 0.163 \\
\hline Utilidad & 0.099 & 0.000 & 0.902 & 0.511 \\
\hline Motivación & 0.054 & 0.056 & 0.553 & 0.972 \\
\hline Confianza & 0.175 & 0.082 & 0.034 & 0.873 \\
\hline
\end{tabular}

Con relación al lugar de procedencia en las puntuaciones, no hay diferencia significativa en los factores agrado, utilidad y motivación debido a que presentan una significancia asintótica mayor que 0.05 , por consiguiente, los departamentos de Lempira, La Paz y El Paraíso se comportan igual. Esto quiere decir que muestran el mismo agrado, utilidad y motivación hacia las matemáticas, sin embargo, el departamento presenta una motivación baja es La Paz, luego El Paraíso y el que presenta una motivación más alta es Lempira. En referencia con el factor confianza sí hay diferencias significativas menor que 0.05. El departamento que muestra mayor confianza es El Paraíso, seguido de Lempira y por último La Paz. Estos resultados pueden darse debido a las metodologías y estrategias didácticas en el proceso de enseñanza-aprendizaje en cada uno de los departamentos.

Considerando el grado que cursan los estudiantes, los factores que presentan significancia asintótica mayor que 0.05 son motivación y confianza, entonces no presentan diferencia significativa; el tercer ciclo de igual manera presenta un alto nivel de confianza y notoria motivación baja. En ambos factores se aprecia que en séptimo grado se presenta mayor motivación y agrado, estos factores van disminuyendo a medida que avanzan a octavo y noveno grado. En cuanto a los factores que presentan diferencia significativa, el curso que tiene mayor agrado y utilidad es séptimo, seguido de octavo y noveno. Se podría suponer que esta diferencia resulte porque aún en séptimo grado no han comenzado a estudiar álgebra. 
En cuanto a la variable cualitativa género, hombres y mujeres presentan igual agrado, utilidad, motivación y confianza, ya que la significancia asintótica es mayor que 0.050. Cabe recalcar que la motivación se encuentra cerca del límite, por lo tanto, se puede inferir que hombres y mujeres tienen una tendencia de comportamiento desigual, la cual llevaría a que las mujeres presentan mayor desmotivación que los hombres.

Los resultados de las pruebas paramétricas de variables dicotómicas como es en el caso de la ansiedad para el género se aplica la prueba t-student, en esta se obtiene una significancia de o.06o. Lo anterior nos indica que hombres y mujeres tienen el mismo nivel de ansiedad hacia las matemáticas. A las variables politómica se aplicó la prueba la prueba ANOVA de un factor, de tal manera que para el lugar de procedencia no se presentan diferencias significativas, o sea que los estudiantes de Lempira, La Paz y El Paraíso muestran el mismo grado de ansiedad. Respecto al grado académico, hay diferencias significativas, el menor nivel de ansiedad se presenta en séptimo grado, seguido de noveno grado y por último octavo grado.

\section{Discusión y conclusiones}

Los resultados obtenidos en esta investigación muestran que la actitud de los estudiantes hacia las matemáticas es rápidamente positiva cercana a la media de 88.89 y una moda de 84 puntos. Estos datos son similares con Pedrosa-Jesús, LeónMantero y Cuida-Gómez (2020), cuyo estudio está enfocado en las actitudes hacia las matemáticas en los grados en educación infantil y primaria, por lo que coinciden que la actitud hacia la matemática es globalmente positiva. Asimismo, los resultados derivados de este estudio son parecidos a los obtenidos por Hidalgo-Alonso, Maroto-Sáez y Palacios-Picos (2004), en la forma en que los estudiantes perciben las matemáticas potenciándola como una disciplina útil, tanto en el ámbito académico como laboral.

Es notable mencionar que en el factor motivación, se obtuvo una media de 8.73 puntos baja con respecto a la media teórica $(\mathrm{MT}=9)$, mostrando que los estudiantes se encuentran desmotivados a pesar de que sienten agrado, utilidad y confianza hacia la asignatura; de una u otra manera la motivación que ellos puedan sentir hacia el estudio de las matemáticas está asociada con su grado de comprensión y su rendimiento en la asignatura. Estas apreciaciones coinciden con Gamboa-Araya y Moreira-Mora (2017), quienes exponen que los estudiantes muestran desmotivación en matemáticas, y que, a pesar de sus esfuerzos en la materia, para muchos de ellos esto no se ve reflejado en sus resultados.

Es importante mencionar que mujeres y hombres presentan igual agrado, motivación, utilización, ansiedad y confianza. Aunque en el factor motivación las mujeres alcanzaron puntuaciones bajas, este resultado se contrasta con el estudio de RojasRojas (2020) donde predominan que los hombres prefieren más a las matemáticas, 
además coinciden con los estudios de Flores y Auzmendi (2018) que comentan que la variable género se comparta de manera homogénea en relación con las actitudes hacia las matemáticas y que no existen diferencias significativas entre los factores actitudinales.

En relación con la variable lugar de procedencia solamente es determinante en el factor confianza por que los estudiantes procedentes de El Paraíso muestran mayor confianza al resolver prácticas matemáticas, este nivel de confianza se debe a las metodologías y estrategias que utilizan en el proceso de enseñanza y aprendizaje los docentes. Además, se puede resaltar que el uso de dispositivos tecnológicos es homogéneo para los estudiantes, por consiguiente, facilita el aprendizaje y las actitudes positivas a las matemáticas. En el caso de los grados académicos, resaltamos que existen diferencias significativas en relación con los factores actitudinales, entonces, es necesario mejorar el proceso de aprendizaje de las matemáticas en todos los grados.

Para finalizar, de este estudio pueden surgir nuevas líneas de investigación relacionadas con la correlación entre actitudes y rendimiento académico en el área de matemáticas, además del diseño, análisis e implementación de estrategias de enseñanza-aprendizaje activas y participativas orientadas a la formación de actitudes positivas hacia esta disciplina.

\section{Lista de referencias}

Aiken L.R. Jr. (1970): Attitudes towards Mathematics. Review of Educational Research, 40, 551-596.

Albert-Gómez, M. J. (2007). La investigación educativa: Claves Teóricas. McGrawHill.https://www.academia.edu/download/59095661/LA_INVESTIGACION_ EDUCATIVA._CLAVES_TEORICAS20190430-128070-a4elay.PDF

Auzmendi, E. (1992). Las actitudes hacia la matemática-estadística en la enseñanzas medias y universitarias. Mensajero.

Bisquerra-Alzina, R. (2012). Metodología de la investigación educativa. La Muralla.

Chamorro-Sady F., Pérez-Henríquez, C, \& Flores-López, W. O. (2018). Actitudes y emociones: pautas para el aprendizaje del idioma inglés en la educación superior intercultural. Revista Electrónica de Conocimientos, Saberes y Prácticas, 1(1). https://doi.org/10.30698/recsp.vii1.5

Fernández-Cézar, R. y Aguirre-Pérez, C. (2010). Actitudes iniciales hacia las matemáticas de los alumnos de grado de magisterio de Educación Primaria: Estudio 
de una situación en el EEES. Unión: revista iberoamericana de educación matemática, (23), 107-116.

Flores-López, W. O. y Auzmendi-Escribano, E. (2015). Análisis de la estructura factorial de una escala de actitud hacia las matemáticas. Aula de encuentro, 1(17). 55-77. https://revistaselectronicas.ujaen.es/index.php/ADE/article/view/2256

Flores-López, W. O. y Auzmendi-Escribano, E. (2018). Actitudes hacia las matemáticas en la enseñanza universitaria y su relación con las variables género y etnia. Profesorado, Revista de Currículum y Formación del Profesorado, 22(3), 231-251. https://doi.org/10.30827/profesorado.v22i3.8000

Gamboa Araya, R., \& Moreira Mora, T. E. (2017). Actitudes y creencias hacia las matemáticas: un estudio comparativo entre estudiantes y profesores. Actualidades Investigativas en Educación, 17(1), 514-559.

García-Soto, Y., Flores-López, W. O., \& Olivar Molina S. (2018). Competencias emocionales en la formación del profesorado de educación secundaria y su relación con las actitudes hacia las matemáticas. Revista Electrónica de Conocimientos, Saberes y Prácticas, 1(2). https://doi.org/10.30698/recsp.v1i2.9

Gil, J., Rodríguez, G. y García, E. (1995). Estadística básica aplicada a las ciencias de la educación. Kronos.

Hart L.E. (1989): Describing the affective domain: saying what we mean. En Affect and mathematical problem solving: A new perspective. D.B. McLeod y V.M. Adams Ediciones. New York: Springler-Verlag, pag. 37- 48.

Hernández-Sampieri, R., Fernández-Collado, C. y Baptista-Lucio, M. (2014). Metodología de la investigación. McGraw-Hill.

Hidalgo-Alonso, S., Maroto-Sáez, A. y Palacios-Picos, A. (2004). ¿Por qué se rechazan las matemáticas? Análisis evolutivo y multivariante de actitudes relevantes hacia las matemáticas. Revista de educación, 334, 75-95.

Pedrosa-Jesús, C., León-Mantero, C. y Cuida-Gómez, M. A. (2020). Estudio de las actitudes hacia las matemáticas en los Grados en Educación Infantil y Primaria. Matemáticas, Educación y Sociedad, 3(3), 18-28.

Rojas-Rojas, J. A. (2020). Estilos de Aprendizaje y Actitudes hacia la Matemática en estudiantes del POLISAL de la UNAN-Managua. Torreón Universitario, 8(23), 37- 47. 
Sabariego, M. (2004). El proceso de investigación. En R. Bisquerra-Alzina, Metodología de la investigación edgucativa (127-163). La Muralla. 